\title{
A rare case of Ovarian ectopic pregnancy with IUD in situ: A case report
}

\author{
Saugat Koirala ${ }^{1}$, Pujan Balla ${ }^{1}$, Sachin Koirala ${ }^{1}$, Ajay Pokhrel ${ }^{1}$, Smriti Pant ${ }^{2}$, and Supriya \\ Paudyal $^{1}$ \\ ${ }^{1}$ Dhaulagiri Hospital \\ ${ }^{2}$ Patan Academy of Health Sciences
}

July 28, 2020

\begin{abstract}
We present a rare case of ectopic pregnancy in a woman using IUD. IUD leads to mild inflammation of uterus, nearby fallopian tube and obstruction in conveyance of ovum. Suspicion of ovarian pregnancy should be considered in a woman using IUD with abdominal pain, vaginal bleeding, positive pregnancy test.
\end{abstract}

\section{Keywords:}

Copper T, Ectopic Pregnancy, Intrauterine device, IUD, Ovarian Pregnancy

\section{Key Clinical Message:}

High index of suspicion of ectopic (much likely ovarian) pregnancy should be considered if a woman with IUD in situ presents with abdominal pain, vaginal bleeding and positive urine pregnancy test.

\section{Introduction:}

Copper $\mathrm{T}$ is a type of Intrauterine Device (IUD), which has a contraceptive failure rate of $0.6 \%$ with perfect use and $0.8 \%$ with typical use [1]. Several risk factors for ectopic pregnancy have been identified which include previous history of ectopic pregnancy, prior tubal surgery, smoking, prior Intrauterine Device (IUD) use, etc. [2]. Women with history of IUD use in past have 16.27 times the risk of Ectopic pregnancy compared with women who have used no contraception [3] For every 1000 live births, about 12 visits to the Emergency Department in the United States have been due to Ectopic pregnancy [4]. Ovarian pregnancy, which occurs when the fertilized ovum gets trapped in the ovary, constitute around three percent of all ectopic pregnancies [5]. We are reporting a case of a woman with intrauterine contraceptive device in situ with ruptured ovarian ectopic pregnancy.

\section{Case:}

A 33-year-old lady with G4P3L3 had presented to Emergency Department (ED) of Dhaulagiri Hospital, Nepal with history of amenorrhea for 43 days and irregular vaginal bleeding in the past 2 weeks with partial soaking of one pad per day. She had abdominal pain and occasional dizziness on the same day of visit to the ED. She also gave history of having regular menstruation cycle in past. Her last child birth was nine years back. She had been using Copper T (Cu T 380A) as a form of contraception since last five years. She gave no history of use of any form of contraception prior to that. She is nonsmoker and gave no history of any medical or surgical illnesses in past.

She was well oriented to time, place and person but looked pale. Her Blood pressure was $110 \mathrm{~mm} \mathrm{Hg}$ systolic and $60 \mathrm{~mm} \mathrm{Hg}$ diastolic and pulse was 108 beats per min. On abdominal examination there was 
generalized tenderness over the suprapubic region with mild distension. Vulva and vagina looked healthy. Copper $\mathrm{T}$ thread was visible on the speculum examination and cervix looked healthy (Figure 1). Her pelvic examination was significant for a firm mobile mass around the left adnexa of $4 \mathrm{~cm} \times 4 \mathrm{~cm}$. Despite the history of contraception, the examination findings led to a strong suspicion towards the differential diagnosis of ectopic pregnancy.

We ordered a urine pregnancy test which came out to be positive. We performed an abdominal sonography on her which showed a well-defined heterogenous lesion of $3.9 \mathrm{~cm} \times 4.2 \mathrm{~cm}$ in the right adnexa overlying the ovary. Ultrasound examination also revealed gross intraperitoneal fluid collection and Copper $\mathrm{T}$ in situ (Figure 2).

We decided to perform exploratory laparotomy on the patient after taking proper informed consent for removal of IUD and the operative procedure. Hemoperitoneum of 1 liter was appreciated. The right ovary size was $4 \mathrm{~cm} \times 5 \mathrm{~cm}$ size with defect of $1 \mathrm{~cm}$ on its surface with firm mass inside and clots overlying it (Figure 3). Bilateral fallopian tubes and left sided ovary were normal. We performed right sided oophorectomy (Figure 4). The IUD string was pulled and the device was removed in the operation theater (Figure 4). Gross examination of the cross-section of the ovary showed fetal tissue with placental component thus confirming ovarian ectopic pregnancy (Figure 5). Her postoperative period was uneventful and she was pleased with overall management at this center. She gave informed consent for the publication of her case report.

\section{Discussion:}

IUD is a top tier contraceptive which decreases the incidence of pregnancy overall [1].Various adverse pregnancy events may occur when IUD is in place namely spontaneous abortion, septic abortion, fetal malformation, preterm labor and chorioamnionitis [6]. Women with history of IUD use in past have significant risk of having ectopic pregnancy compared with women who used no contraception [3]. Copper T and Levonorgesterel containing IUD are modern generation of IUD with ectopic pregnancy rates of 0.2 per 1,000 woman-year [7]. Then again, when we consider the possibility of ectopic pregnancy with the current use of IUD, literature has limited answer.

A study, with 287 cases with ectopic pregnancy, done by Pagano had shown 40 patients had IUD in situ [8]. Among them, 80.5\% used Lippes Loop as IUD [8]. However, given the differences between Lippes Loop and the IUD used at present, it may not be suitable to draw conclusions regarding the relationship between current IUDs and the risk of ectopic pregnancy.

Raziel and colleagues found a strong association of ovarian pregnancy and IUD in situ. Out of 19 diagnosed with ovarian ectopic pregnancy, 13 women (68\%) had been using IUD during diagnosis [9]. But then, this study doesn't mention the type of IUD or if it was lying misplaced [9].

Ercal et al described two cases where ovarian pregnancy was associated with IUD use [10]. However, both the cases don't qualify for concurrent use of IUD and ovarian pregnancy. While the first case had the IUD removed one month prior to the diagnosis of ovarian pregnancy, the other woman had used Lippes Loop for almost 16 years [10].

Similar to our study, Annaiah and colleagues had reported a case of ovarian ectopic pregnancy with IUD in situ [11]. In their case, the patient was hemodynamically stable and was managed laparoscopically [11]. The patient in our study was tachycardic and ultrasonography revealed features of gross collection intraperitoneally and was managed with laparotomy. Another case of primary ovarian ectopic pregnancy with IUD in situ was managed with laparotomy, wedge reconstruction and repair of the ovary [12]. The defect was limited on the superficial surface which was managed with reconstructive procedures in that case. On the contrary, in our case the defect was deep and fetal tissue invaded the ovary. This had prompted for removal of the ovary in our scenario. Findings of tubal ectopic pregnancy with IUD in situ was seen in another case report by Neth et al [13]. Another reported case of ovarian ectopic pregnancy with concurrent use of IUD encourages the use of sonography in women with abdominal pain, vaginal bleeding and positive urine pregnancy tests [14]. The diagnosis of ectopic pregnancy will not be missed by this approach. 
The cellular and humoral components of IUD generate inflammatory reaction of the endometrium. These elements are expressed at the tissue and fluid content of the uterine cavity which prevents fertilization [15]. The uterine-tubal junction patency in human allows the passage of air and fluid contents from uterine cavity to the tubal lumen. Thus, the Copper ions in the tube is increased to similar amounts to that in uterine fluid [16]. This suggests that aside from preventing uterine pregnancy IUD also protects against tubal ectopic pregnancy.

Most case studies in women with ectopic pregnancy and IUD in situ are invariably ovarian pregnancy [9-12, 14]. With these evidences, it looks necessary to further study the association of IUD and Ovarian pregnancy and thus highlight the concept of 'IUD Associated Ovarian Pregnancy Phenomenon'. The idea is, IUD leads to mild inflammation of the uterus and nearby fallopian tube and also may lead to obstruction in ovum conveyance [17]. This property of IUD would be responsible for preventive action of it on pregnancy (both on the uterine cavity and the fallopian tube). Additionally, as ovary is devoid of inflammatory action of the device, when ectopic pregnancy occur, ovary becomes the most likely site of pregnancy.

A long-term study is required to prove this hypothesis and draw a conclusion to the statement. Nevertheless, limiting factor to conduct this kind of research would be the rarity of pregnancy conceived with IUD and infrequency of ovarian pregnancy overall. Yet, high index of suspicion of ovarian pregnancy should be considered if a woman with IUD in situ presents with abdominal pain, vaginal bleeding and positive urine pregnancy test.

\section{Contributors:}

SK1, AP and SP2 were involved in diagnosing the patient. SK1, PB and SK2 were involved in treatment and management of the patient (wherein SK1 was the leading surgeon; PB and SK2 were the anesthesiologists in charge). SK1 and SP1 wrote majority of the manuscript and formulated the hypothesis. All co-authors provided critical feedback and helped shape the research, analysis and manuscript.

\section{Declaration of Competing Interest}

The authors declare that they have no conflict of interest regarding the publication of this case report.

\section{Funding}

No specific grant from any funding agency supported the publication of this case report.

\section{Ethical approval}

As this was a case report ethical approval from Institutional Review Board was not sought. However, written informed consent was obtained from the patient.

\section{Patient consent}

Written consent for publication was obtained from the patient.

Acknowledgements: We thank the patient and her husband in co-operating with the history taking and the nursing staff in managing the patient during the hospital stay. We would also like to thank Dr. Dipti Das for providing valuable feedback in structuring the manuscript.

\section{References:}

1. Trussell, J., Contraceptive failure in the United States.Contraception, 2011.83 (5): p. 397-404.

2. Bouyer, J., et al., Risk factors for ectopic pregnancy: a comprehensive analysis based on a large case-control, population-based study in France. American journal of epidemiology, 2003.157 (3): p. 185-194. 
3. Gaskins, A.J., et al., Demographic, lifestyle, and reproductive risk factors for ectopic pregnancy. Fertil Steril, 2018.110 (7): p. 1328-1337.

4. Mann, L.M., et al., Trends in Ectopic Pregnancy Diagnoses in United States Emergency Departments, 2006-2013. Matern Child Health J, 2020. 24 (2): p. 213-221.

5. Chukus, A., et al., Uncommon implantation sites of ectopic pregnancy: thinking beyond the complex adnexal mass. Radiographics, 2015. 35 (3): p. 946-959.

6. Brahmi, D., et al., Pregnancy outcomes with an IUD in situ: a systematic review. Contraception, 2012. 85 (2): p. 131-139.

7. Franks, A.L., et al., Contraception and ectopic pregnancy risk. American journal of obstetrics and gynecology, 1990.163 (4): p. 1120-1123.

8. Pagano, R., Ectopic pregnancy: A seven-year survey. Medical Journal of Australia, 1981. 2 (11): p. 586-588.

9. Raziel, A., et al., Ovarian pregnancy-a 12-year experience of 19 cases in one institution. European Journal of Obstetrics \& Gynecology and Reproductive Biology, 2004. 114 (1): p. 92-96.

10. Ercal, T., et al., Ovarian pregnancy; relationship to an intrauterine device. Australian and New Zealand journal of obstetrics and gynaecology, 1997. 37 (3): p. 362-364.

11. Annaiah, T. and J. Gemmell, Ovarian ectopic pregnancy with IUD in situ managed by conservative laparoscopic surgery using the LigaSure V device. Journal of Obstetrics and Gynaecology, 2009.29 (3): p. 266-268.

12. Tantuway, B., et al., Primary Ovarian Ectopic Pregnancy.Journal of Case Reports, 2017.7 (2): p. 130-132.

13. Neth, M.R., et al., Ruptured Ectopic Pregnancy in the Presence of an Intrauterine Device. Clin Pract Cases Emerg Med, 2019.3 (1): p. 51-54.

14. Smith, G., Ovarian pregnancy associated with a copper-7 intrauterine device: report of a case and review of the literature. The Journal of the American Osteopathic Association, 1982. 81 (11): p. 796.

15. Ortiz, M.E. and H.B. Croxatto, Copper-T intrauterine device and levonorgestrel intrauterine system: biological bases of their mechanism of action. Contraception, 2007. 75 (6): p. S16-S30.

16. Larsson, B., B. Ljung, and L. Hamberger, The influence of copper on the in vitro motility of the human Fallopian tube. American journal of obstetrics and gynecology, 1976. 125 (5): p. 682-690.

17. Herbertsson, G., S.S. Magnusson, and K. Benediktsdottir, Ovarian pregnancy and IUCD use in a defined complete population.Acta obstetricia et gynecologica Scandinavica, 1987. 66 (7): p. 607-610.

Figures

Figure 1: Copper $\mathbf{T}$ thread visualized in speculum examination coming from external os

Figure 2: Ultrasonography image showing copper $\mathrm{T}$ in situ

Figure 3: Intraoperative finding of Ovarian ectopic

Figure 4: Removed specimen of ovary and copper $T$ with placental bits from rupture site

Figure 5: Gross cross section examination of ovary demonstrating fetal part inside ovary 

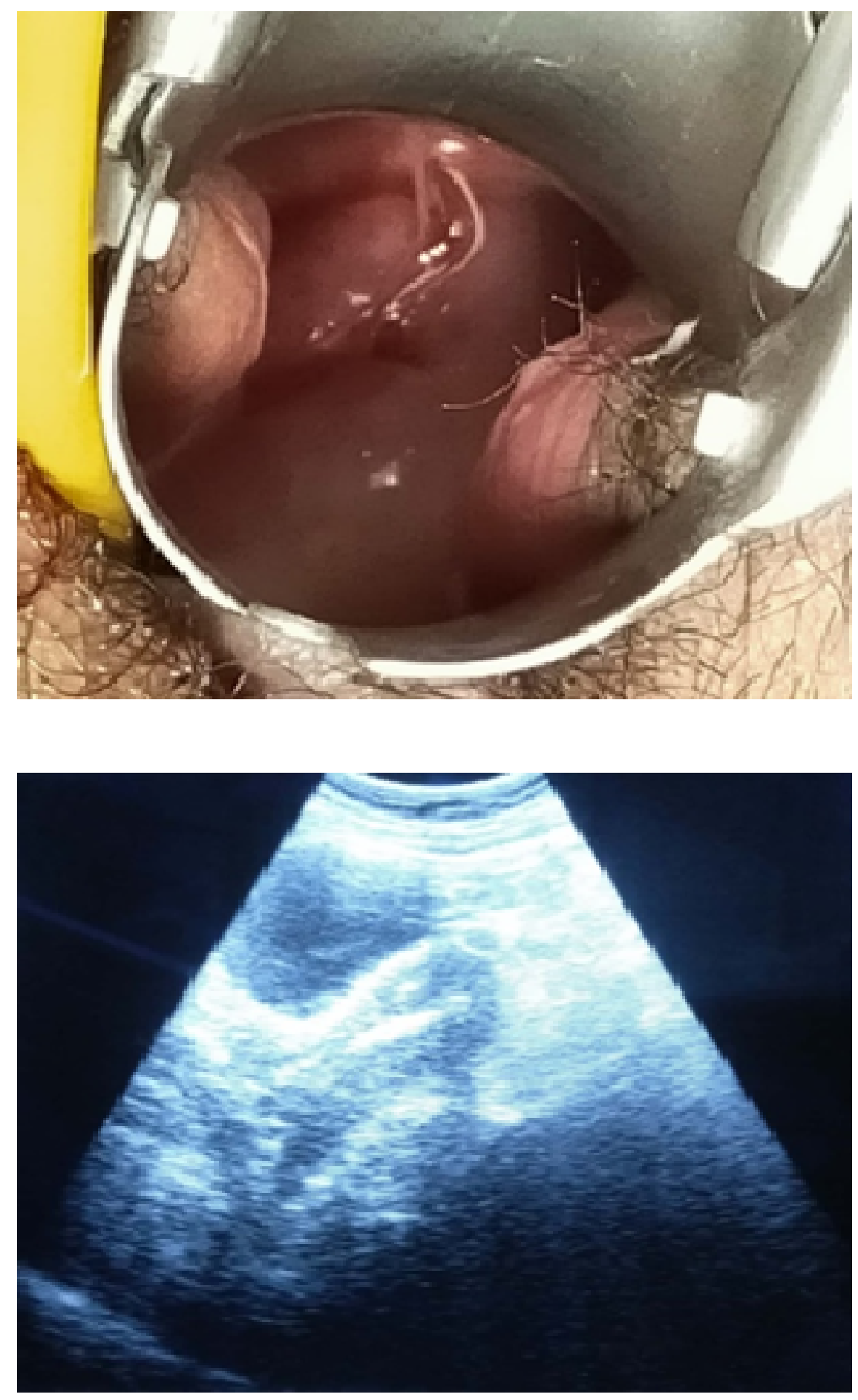

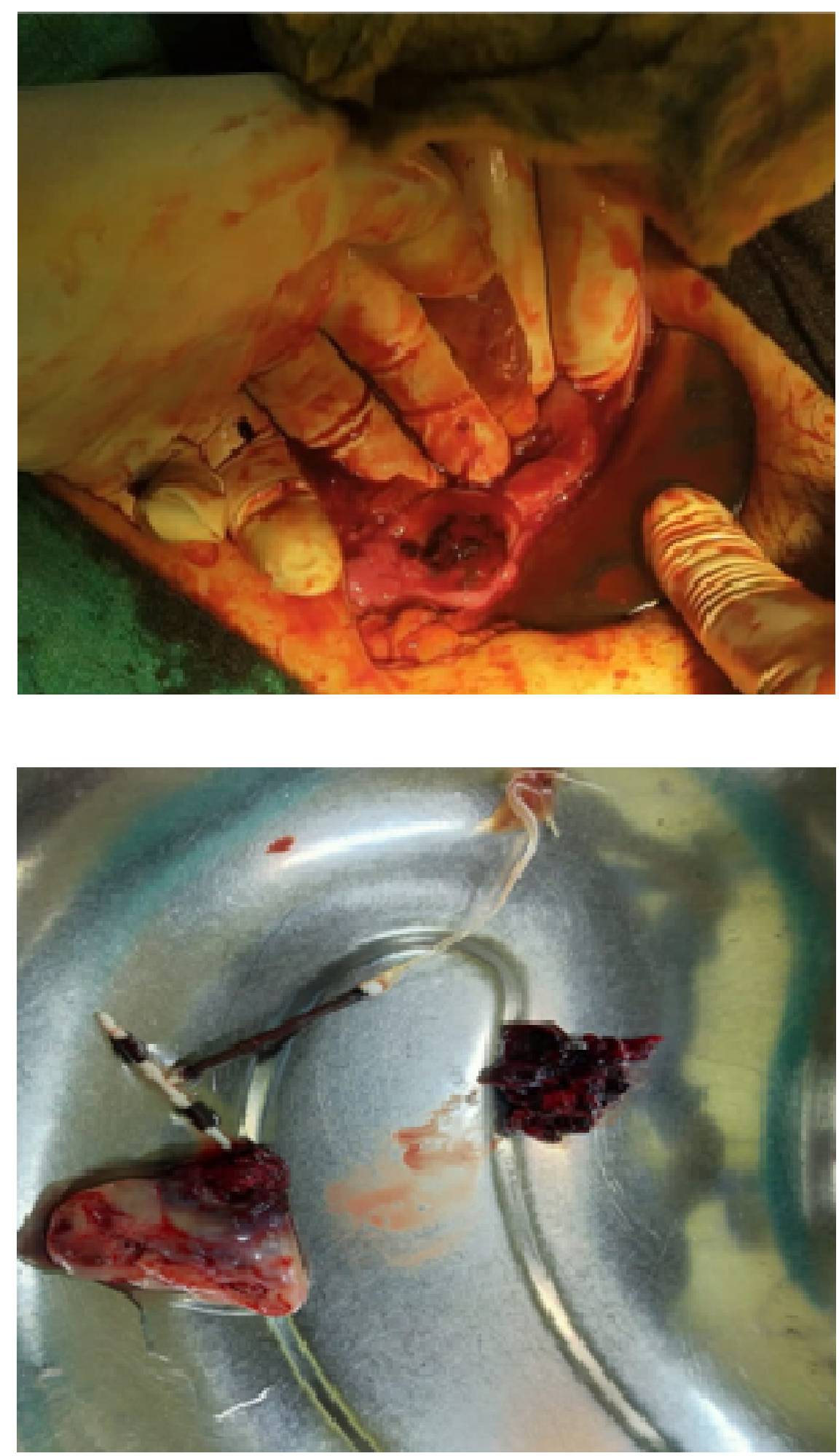


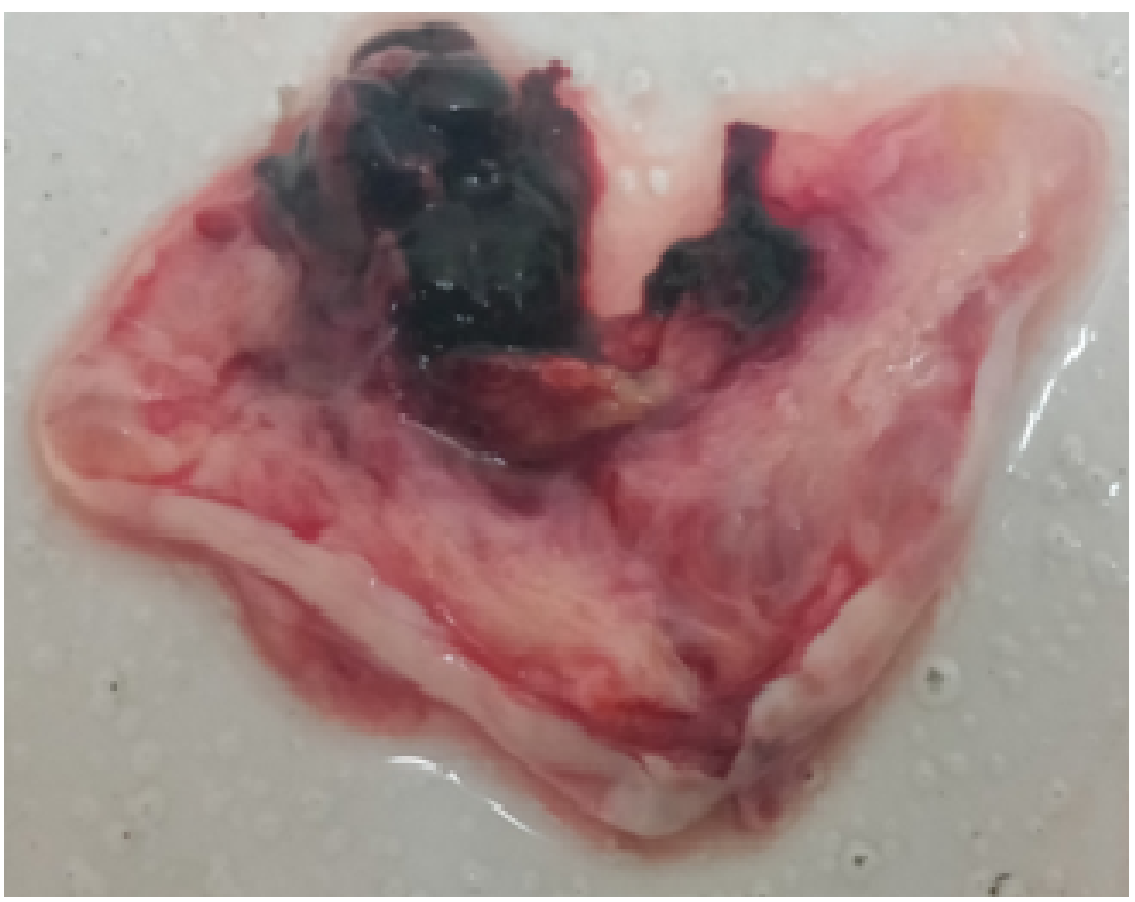

